\title{
An Updated Host Differential Due to Two Novel Races of Diplocarpon rosae Wolf, the Causal Agent of Rose Black Spot Disease
}

\author{
David C. Zlesak \\ University of Wisconsin-River Falls, 410 S. 3rd Street, River Falls, WI 54022
}

Darcy Ballantyne, Matthew Holen, Andrea Clark, and Stan C. Hokanson University of Minnesota, Department of Horticultural Science, 1970 Folwell Avenue, St. Paul, MN 55108

Kristen Smith

Star ${ }^{\circledR}$ Roses and Plants, 8 Federal Road Suite 6, West Grove, PA 19390

Jason D. Zurn and Nahla V. Bassil

USDA-ARS National Clonal Germplasm Repository, 33447 Peoria Road, Corvallis, OR 97333

\section{James M. Bradeen}

University of Minnesota, Department of Plant Pathology, 1991 Upper Buford Circle, St. Paul, MN 55108

Additional index words. defoliation, disease resistance, Marssonina rosae, Rosa, virulence

\begin{abstract}
The fungal pathogen, Diplocarpon rosae, infects only roses (Rosa spp.) and leads to rose black spot disease. Rose black spot is the most problematic disease of outdoorgrown roses worldwide due to the potential for rapid leaf chlorosis and defoliation. Eleven races of the pathogen were previously characterized from isolates collected in North America and Europe. Isolates of $D$. rosae obtained from infected leaves of the roses Brite Eyes ${ }^{\text {TM }}$ ('RADbrite'; isolate BEP; collected in West Grove, PA) and Oso Easy ${ }^{\circledR}$ Paprika ('CHEwmaytime'; isolate PAP; collected in Minneapolis, MN) proved to have unique infection patterns using the established host differential with the addition of Lemon Fizz $^{\mathrm{TM}}$ ('KORlem'). The new races are designated race 12 (BEP) and race 13 (PAP), respectively, and Lemon Fizz ${ }^{\mathrm{TM}}$ should be included in the updated host differential because it distinguishes races 7 and 12. Additionally, inconsistent infections and limited sporulation were found in the host differential Knock Out ${ }^{\circledR}$ ('RADrazz') for races 7 and 12. Expanding the collection of $D$. rosae races supports ongoing research efforts, including host resistance gene discovery and breeding new rose cultivars with increased and potentially durable resistance.
\end{abstract}

Rose black spot disease is caused by the hemi-biotrophic fungus Diplocarpon rosae, and this pathogen solely infects roses (Debener, 2019). Rose black spot is considered the most problematic and widespread disease of outdoor-grown roses in humid/wet

Received for publication 3 Feb. 2020. Accepted for publication 31 July 2020.

Published online 23 September 2020

This work was funded by the U.S. Department of Agriculture/National Institute of Food and Agriculture Specialty Crop Research Initiative project "RosBREED: Combining Disease Resistance with Horticultural Quality in New Rosaceous Cultivars" under award no. 2014-51181-22378.

We thank Bailey Nurseries and Star ${ }^{\circledR}$ Roses and Plants for the donation of roses.

S.C.H. is the corresponding author. E-mail: hokan017@ umn.edu.

This is an open access article distributed under the CC BY-NC-ND license (https://creativecommons. org/licenses/by-nc-nd/4.0/). the continuation of the asexual stage the following growing season.

Isolates of $D$. rosae have been collected and preserved by growing them on fresh leaf tissue of susceptible host cultivars or culturing them on artificial media (Whitaker et al., 2007b). Single spore isolates are commonly prepared to ensure each isolate is a single genotype. Over the years, multiple research groups have collected isolates within their geographic region and classified them into physiological races based on virulence on a set of independently chosen host cultivars (Bolton and Svejda, 1979; Debener et al., 1998; Whitaker et al., 2007b; Yokoya et al., 2000). Unfortunately, many of these isolates have not been effectively preserved and are no longer available.

Whitaker et al. (2010b) obtained and further characterized isolates of $D$. rosae that had been classified into races by researchers in North America and Europe with the goal of establishing and preserving an international race collection with unified nomenclature. Isolates from these different research groups were resolved into 11 unique races and designated races 1 to 11 . The international $D$. rosae isolate collection is cryopreserved at the University of Minnesota, and isolates have been made available to researchers on request and with appropriate permits.

Characterized isolates of $D$. rosae have been useful for multiple research applications. Practical applications of characterized $D$. rosae isolates have included studying geographical race distribution (Werlemark et al., 2006; Whitaker et al., 2007c), characterizing race-specific and nonrace-specific resistance in specific rose cultivars (Zlesak et al., 2010), and the discovery and characterization of resistance genes (von Malek and Debener, 1998; Whitaker et al., 2010a; Zurn et al., 2018, 2020).

Identifying and adding unique races to the international $D$. rosae race collection strengthens this valuable resource. Characterizing isolates obtained from cultivars that have not previously displayed black spot symptoms in a region (resistance-breaking isolates) is one method of streamlining the identification of possible new races. Such isolates may result from the migration of a previously characterized race to a new region or may represent a new race that may have occurred through sexual reproduction or mutation. Moreover, the identification of pathogen races within a region can provide new insights to breeders and growers regarding resistance gene deployment and stewardship.

\section{Materials and Methods}

, common with rain and dew events) Additionally, spores typically spread to uninfected tissue through rain splash.

The life cycle of $D$. rosae includes an asexual stage (which predominates) and a sexual stage that is seldom observed (Debener, 2019). Repeated infection of recently emerged foliage of susceptible cultivars can occur throughout the growing season. Conidia overwinter on leaf debris and stem tissue and serve as inoculum for 
collected in eastern North America) (Zlesak et al., 2010). The authors had not observed and did not have knowledge of these cultivars previously displaying black spot symptoms in north central and northeastern landscapes in the United States. A single spore isolate from infected leaves of Brite Eyes ${ }^{\mathrm{TM}}$ and Oso Easy ${ }^{\circledR}$ Paprika were cultured and the isolates were designated BEP and PAP, respectively. These isolates have been maintained using detached leaves of the cultivars from which they were collected and also 'Morden Blush', a cultivar susceptible to all $D$. rosae races in the collection. The isolates were also cryopreserved on leaf tissue and accessed again, as needed, according to Whitaker et al. (2007a).

Nine cultivars in the $D$. rosae international host differential (Whitaker et al., 2010b) (the first nine cultivars in Table 1) plus 'Morden Blush' as a universal positive control were challenged with isolates BEP and PAP using detached leaf assays. Additionally, the roses Brite Eyes ${ }^{\mathrm{TM}}$, Oso Easy ${ }^{\circledR}$ Paprika, and Lemon Fizz ${ }^{\mathrm{TM}}$ ('KORfizzlem') were each challenged with races 1 to 11 and BEP and PAP. Knock Out ${ }^{\circledR}$ ('RADrazz') was also challenged with isolate $\mathrm{R} 6$ of race 7 to confirm the report by Rouet et al. (2019) that Knock Out ${ }^{\circledR}$ is susceptible to race 7, which differed from a previous report (Whitaker et al., 2010b). Inoculations were conducted as previously described (Whitaker et al., $2010 \mathrm{~b}$ ), with the modification of pipetting inoculum onto leaves instead of misting to have more controlled inoculum placement as described by Zurn et al. (2018). To avoid cross-contamination of isolates, sterile, disposable $50-\mathrm{mL}$ centrifuge tubes and pipettes were used to prepare and distribute inoculum and new nitrile gloves were used between handling each isolate. Reactions were scored as susceptible if expanding lesions with acervuli were present at or before day 14 after inoculation and resistant if at day 14 acervuli were not present when viewed under a dissecting microscope.

At least four boxes per differential hostisolate (BEP and PAP) combination were prepared, and each box had at least two leaves. Two boxes of each differential host- isolate (BEP and PAP) combination (along with two boxes of 'Morden Blush' per each isolate as a positive control and Brite $\mathrm{Eyes}^{\mathrm{TM}}$ and Lemon Fizz ${ }^{\mathrm{TM}}$ ) were inoculated on 15 Mar. 2020 and assessed on 29 Mar. 2020. Inoculated boxes were grouped on a separate section of the laboratory bench based on the isolate and then arranged within groups using a completely randomized design. The remaining two or more boxes of leaves for each differential host-isolate (BEP and PAP) combination were inoculated during a 3-year period (Apr. 2016 to Dec. 2019) coinciding with routine race/isolate maintenance. The 11 races and BEP and PAP were grown continually on leaf tissue with inoculum prepared and transferred to fresh leaves approximately every 2 weeks. 'Morden Blush' was one of the susceptible hosts used for isolate maintenance, thus allowing it to serve consistently as the positive control. Three boxes (with two or more leaves) from susceptible host(s) (at least one box of which was leaves of 'Morden Blush') were prepared for each isolate as part of isolate maintenance. Simultaneously, the boxes containing leaves from the diagnostic clones were inoculated, and boxes were prepared and grouped in a unique area of the laboratory bench by isolate to prevent crosscontamination. Additionally, subsets of host differential cultivars that distinguished specific races were periodically challenged during race maintenance for added assurance that the isolates still had the reactions expected and cross-contamination did not occur.

Coinciding with race maintenance during the same 3-year period, at least four boxes (with at least two leaves each) of the following combinations were also inoculated: Brite Eyes $^{\mathrm{TM}}$, Oso Easy ${ }^{\circledR}$ Paprika, and Lemon Fizz $^{\mathrm{TM}}$ were inoculated with the 11 races and BEP and PAP and Knock Out ${ }^{\circledR}$ was inoculated with race 7 .

\section{Results}

Resistant and susceptible reactions for the host-isolate combinations were generally consistent across replications and straightforward to score, with no observed evidence of cross-contamination of isolates/races. Sus- ceptible cultivars had multiple sporulating lesions develop per leaf, and resistant cultivars did not have observable lesions. The reactions of Knock Out ${ }^{\circledR}$ with isolates BEP and R6 (race 7) were exceptions. There were inconsistencies in the occurrences of infection and lesion growth on Knock Out ${ }^{\circledR}$ for the different boxes of leaves for each of the isolates. Due to these inconsistencies, the boxes for each inoculation date for Knock Out $^{\circledR}$ were observed until day 25 to note whether additional leaves developed lesions and to watch for further development of the lesions that did develop. No additional lesions developed other than those visible by day 14 , and sporulating lesions that did start by day 14 did not noticeably continue to expand after day 14.

BEP and R6 (race 7) shared the same infection pattern on the original host differential (Table 1). However, adding the rose Lemon Fizz ${ }^{\mathrm{TM}}$ to the differential distinguished them as separate races; Lemon Fizz $^{\mathrm{TM}}$ was susceptible to race 7 and resistant to BEP. Therefore, BEP represents a newly identified race of D. rosae and is designated as race 12. PAP had a unique infection pattern on the original host differential and represents a newly identified race of $D$. rosae designated race 13

Oso Easy ${ }^{\circledR}$ Paprika shared the same race infection pattern across the 13 races as the host differential rose Baby Love ${ }^{\mathrm{TM}}$ ('SCRivluv'). Both roses were susceptible to races 11 and 13 and resistant to all the other races. Interestingly, Brite Eyes $^{\mathrm{TM}}$ and Lemon Fizz $^{\mathrm{TM}}$ were each susceptible to only a single race in the international race collection (race 12 and race 7 , respectively).

\section{Discussion}

Isolates BEP and PAP represent two newly identified races of $D$. rosae, and inclusion of them in the international $D$. rosae race/isolate collection helps to strengthen and expand this important resource. Lemon Fizz $^{\mathrm{TM}}$ was critical to distinguishing races 7 (isolate R6) and 12 (isolate BEP), thereby justifying its inclusion in the $D$. rosae international host differential (Zlesak et al.,

Table 1. Expanded rose black spot host differential set. Disease responses are labeled as susceptible $(+)$ or resistant $(-)$.

\begin{tabular}{|c|c|c|c|c|c|c|c|c|c|c|c|c|c|}
\hline \multirow[b]{3}{*}{ Host $^{2}$} & \multicolumn{13}{|c|}{ Race } \\
\hline & 1 & 2 & 3 & 4 & 5 & 6 & 7 & 8 & 9 & 10 & 11 & 12 & 13 \\
\hline & HSN & 2402 E1 & GVH & Dü A3 & B 005 & Dort E4 & R6 & $\mathrm{ACT}$ & IGWA & KOMN & CW1 & BEP & PAP \\
\hline Honeybee $^{\mathrm{TM}}$ ('ZLEhoney') & - & - & + & + & + & + & + & - & - & + & - & + & + \\
\hline Sexy Rexy ${ }^{\circledR}$ ('MACrexy’) & - & - & - & + & + & + & + & + & + & + & + & + & + \\
\hline Surrey ('KORlanum') & - & - & - & - & - & + & + & + & + & + & + & + & + \\
\hline Love and Peace ${ }^{\mathrm{TM}}$ ('BAIpeace') & - & - & - & - & - & - & + & + & + & + & + & + & + \\
\hline Baby Love ${ }^{\mathrm{TM}}$ ('SCRivluv') & - & - & - & - & - & - & - & - & - & - & + & - & + \\
\hline 'Hansa' & + & - & - & - & - & - & - & - & - & - & - & - & - \\
\hline Lemon Fizz ${ }^{\mathrm{TM}}$ ('KORfizzlem’) & - & - & - & - & - & - & + & - & - & - & - & - & - \\
\hline
\end{tabular}

${ }^{\mathrm{z}}$ Cultivar name or trade name with the cultivar name in parentheses.

${ }^{\mathrm{y}}$ Knock Out ${ }^{\circledR}$ was originally scored as resistant to isolate R6 (race 7) by Whitaker et al. (2010b); however, it was found to be inconsistently susceptible in this study and susceptible by Rouet et al. (2019). Knock Out ${ }^{\circledR}$ displayed a similarly inconsistent susceptible reaction with BEP (race 12) in this study. 
2017). As demonstrated in this study, as more hosts are added to a differential set, there is the possibility of discovering hosts that can separate isolates previously grouped as a common physiologic race into different races (Debener, 2019). A small differential set that does not produce robust phenotypic results risks underestimating the number of races from a collection of isolates.

Rouet et al., (2019) found that Knock Out ${ }^{\circledR}$ was susceptible to race 7 (isolate R6), in contrast to the results of Whitaker et al. (2010b), who reported it to be resistant. In an attempt to address this discrepancy, we challenged eight boxes of leaves of Knock Out ${ }^{\circledR}$ over three separate dates with the same isolate of race 7 as reported by Rouet et al. (2019). Interestingly, the reaction was inconsistent over the inoculation dates and between replications within dates. We found similar inconsistencies between Knock Out ${ }^{\circledR}$ and BEP, with only some leaves developing sporulating lesions; the lesions that did develop were relatively small and had limited acervuli. These discrepancies may be due, in part, to the relatively strong partial black spot resistance documented in Knock Out ${ }^{\circledR}$. When Knock Out ${ }^{\circledR}$ is infected in landscape settings, black spot development occurs much more slowly than it does on other susceptible cultivars (Colbaugh et al., 2005). Whitaker et al. (2010b) reported that for the races Knock Out ${ }^{\circledR}$ was susceptible to, lesions typically stopped expanding in size by day 14 after inoculation. In our experience, isolate R6 has been a relatively slower- and weakergrowing isolate across susceptible hosts, which may contribute to the inconsistencies in infection when placed on Knock Out ${ }^{\circledR}$. Additionally, continually growing the isolates on rose leaves for years may have strengthened the virulence of R6. Regardless of the reason for inconsistent infections of some races on Knock Out ${ }^{\circledR}$, our results support those of Rouet et al. (2019), who reported that Knock Out ${ }^{\circledR}$ should be designated as susceptible to race 7 because sporulating lesions are frequently possible. However, we caution that false-negative results may periodically occur using Knock Out ${ }^{\circledR}$ due to inconsistencies in infection incidence and lesion development; these inconsistencies may be more problematic with some isolates than with others. More work is warranted to gain a better understanding of the resistance mechanism(s) of Knock Out ${ }^{\circledR}$; because of those results, removing it from the host differential should be considered. Fortunately, the other cultivars in the host differential have repeatable and distinct infection reactions.

Rouet et al. (2019) recently identified $D$. rosae isolate VSKO4 as a unique race due to its infection pattern on the international host differential and tentatively named it race 14 . Calling this isolate race 14 is warranted because it is different from race 12 in that 'George Vancouver', Knock Out ${ }^{\circledR}$, and Baby Love $^{\mathrm{TM}}$ are resistant to race 12 and susceptible to race 14. It is also distinguished from race 13 (PAP) in that 'George Vancouver' is susceptible to race 14 and resistant to race 13.

Including $D$. rosae isolates classified as races 12 and 13 of $D$. rosae in the international isolate collection and including Lemon Fizz $^{\mathrm{TM}}$ in the differential host set will expand the usefulness of these resources for the scientific community. Efforts are ongoing to identify and characterize isolates potentially representing new races from numerous research teams and to expand the host differential set as warranted. The primary use of the international race collection to date has been host resistance gene discovery, characterization, and marker development (Terefe and Debener, 2011; Zlesak et al., 2017; Zurn et al., 2018, 2020). Such markers can be used in marker-assisted parental and seedling selection (MAPS and MASS) approaches to develop roses with durable resistance to rose black spot disease.

\section{Literature Cited}

Bolton, A.T. and F.J. Svejda. 1979. New race of Diplocarpon rosae capable of causing severe black spot on Rosa rugosa hybrids. Can. Plant Dis. Surv. 59:38.

Colbaugh, P.F., W.T. Crow, W.A. Mackay, and S.W. George. 2005. Varietal reaction of selected rose varieties to black spot. Biol. and Cultural Tests for Control of Plant Dis. 20:17.

Debener, T. 2019. The beast and the beauty: What do we know about black spot in roses? Crit. Rev. Plant Sci. 38(4):313-326.

Debener, T., R. Drewes Alvarez, and K. Rockstroh. 1998. Identification of five physiological races of blackspot, Diplocarpon rosae Wolf on roses. Plant Breed. 117:267-270.

Rouet, C., E. Lee, T. Banks, J. O’Neill, R. LeBlanc, and D. Somers. 2019. Identification of a polymorphism within the Rosa multiflora muRdr1A gene linked to resistance to multiple races of Diplocarpon rosae $\mathrm{W}$. in tetraploid garden roses $($ Rosa $\times$ hybrida). Theor. Appl. Genet. 133(1):103-117.

Terefe, D. and T. Debener. 2011. An SSR from the leucine-rich repeat region of the rose $R d r l$ gene family is a useful resistance gene analogue marker for roses and other Rosaceae. Plant Breed. 130:291-293. von Malek, B. and T. Debener. 1998. Genetic analysis of resistance to blackspot (Diplocarpon rosae) in tetraploid roses. Theor. Appl. Genet. 96:228-231.

Werlemark, G., B.U. Carlson-Nilsson, and C.G. Davidson. 2006. Genetic variation in the rose pathogen Marssonina rosae estimated by RAPD. Intl. J. Hort. Sci. 12:63-67.

Whitaker, V.M., J.M. Bradeen, T. Debener, A. Biber, and S.C. Hokanson. 2010a. Rdr3, a novel locus conferring black spot disease resistance in tetraploid rose: Genetic analysis, LRR profiling, and SCAR marker development. Theor. Appl. Genet. 120:573-585.

Whitaker, V.M., K. Zuzek, S.C. Hokanson, and J. Bradeen. 2007a. Culturing and long-term storage of virulent races of the rose blackspot pathogen, Diplocarpon rosae. Wolf. Acta Hort 751:199-206.

Whitaker, V.M., K. Zuzek, S.C. Hokanson, and J. Bradeen. 2007b. Resistance of 12 rose genotypes to 14 isolates of Diplocarpon rosae Wolf (rose blackspot) collected from eastern North America. Plant Breed. 126:83-88.

Whitaker, V.M., S.C. Hokanson, and J. Bradeen. 2007c. Distribution of rose black spot (Diplocarpon rosae) genetic diversity in eastern North America using amplified fragment length polymorphism and implications for resistance screening. J. Amer. Soc. Hort. Sci. 132:534-540.

Whitaker, V.M., T. Debener, A.V. Roberts, and S.C. Hokanson. 2010b. A standard set of host differentials and unified nomenclature for an international collection of Diplocarpon rosae races. Plant Pathol. 59:745-752.

Yokoya, K., K.I. Kandasamy, S. Walker, Z. Mandegaran, and A.V. Roberts. 2000. Resistance of roses to pathotypes of Diplocarpon rosae. Ann. Appl. Biol. 136:15-20.

Zlesak, D.C., V.M. Whitaker, S. George, and S.C. Hokanson. 2010. Evaluation of Roses from the Earth-Kind ${ }^{\circledR}$ trials: Black spot (Diplocarpon rosae Wolf) resistance and ploidy. HortScience 45:1779-1787.

Zlesak, D.C., J. Bradeen, S. Kummeth, N. Bassil, J.D. Zurn, and S.C. Hokanson. 2017. Rose parental characterization and population development for the identification of novel rose black spot resistance genes. Proc. VII Intl. Symposium Rose Res. and Cultiv., Angers, 2-7 July 2017 (VII Intl. Symposium Rose Res. and Cultiv. Abstr. S1_P10).

Zurn, J.D., D.C. Zlesak, M. Holen, J.M. Bradeen, S.C. Hokanson, and N.V. Bassil. 2018. Mapping a novel black spot resistance locus in the climbing rose Brite Eyes ${ }^{\mathrm{TM}}$ ('RADbrite'). Front. Plant Sci. 9:1730.

Zurn, J.D., D.C. Zlesak, M. Holen, J.M. Bradeen, S.C. Hokanson, and N.V. Bassil. 2020. Mapping the black spot resistance locus $R d r 3$ in the shrub rose 'George Vancouver' allows for the development of improved diagnostic markers for DNA informed breeding. Theor. Appl. Genet. 133:2011-2020. 